(B)

$$
{ }_{R} \sum_{X} S(X, R) \subset\left(S_{0}, \epsilon R\right) S(X, R)
$$

and $\sum$ is an m-plane through $P$ such that

$$
\left(\sum, \epsilon R_{0}\right) \supset S_{0} \text {. }
$$

Then if $\epsilon \leqq 2^{-2000 N^{2}}$ there will exist a topological $m$-disk $\bar{S}$ such that

$$
S_{0} S\left(P, \frac{1}{16} R_{0}\right) \subset \bar{S} \subset S_{0} S\left(P, R_{0}\right) .
$$

Where $S(x, r)$ is a solid ball of centre $x$ and radius $r$ while $(y, \delta)$ is the set of points lying within $\delta$ of the set $y$.

Oregon State College and

UNiversity of Bristol, ENGLAND

\title{
A CHARACTERIZATION OF THE ALGEBRA OF ALL CONTINUOUS FUNCTIONS ON A COMPACT HAUSDORFF SPACE
}

BY YITZHAK KATZNELSON

Communicated by Walter Rudin, March 23, 1960

This note is a complement to [1]. We consider a commutative, semi-simple and self-adjoint Banach algebra $B$ and assume that $B$ has a unit element and is regular. By $\mathfrak{M}$ we denote the space of maximal ideals of $B$ and, applying the Gelfand representation, we consider $B$ as an algebra of continuous functions defined on $\mathfrak{M}$. It is obvious that if $B$ is $C(\mathfrak{M})$ (the algebra of all the continuous functions on $\mathfrak{M})$ the idempotents in any quotient algebra of $B$ are always bounded. We prove here that this property characterizes $C(\mathfrak{M})$ and give an application of this result.

Lemma 1. Suppose that there exist constants $K$ and $K_{1}, K_{1}<1$ such that to any real, (resp. non-negative) function $f \in C(\mathfrak{M})$ there exists an element $f_{1} \in B$ such that $\| f_{1}\left|\leqq K \operatorname{Sup}_{M \in \mathfrak{M}}\right| f(M) \mid, f-f_{1}$ is real (nonnegative) and

$$
\operatorname{Sup}_{M \in \mathfrak{M}}\left|f(M)-f_{1}(M)\right|<K_{1} \operatorname{Sup}_{M \in \mathfrak{M}}|f(M)| ;
$$

then $B=C(\mathfrak{M})$ and for any $f \in B\|f\| \leqq 4 K\left(1-K_{1}\right)^{-1} \operatorname{Sup}_{M \in \mathfrak{M}}|f(M)|$.

Proof. Define by induction $f_{n}=\left(f-\sum^{n-1} f_{i}\right)_{1}$; then $f=\sum_{1}^{\infty} f_{n}$. 
Lemma 2. Suppose that there exists a constant $K_{2}$ such that if $h$ is an idempotent in any quotient algebra of $B,\|h\|<K_{2}$; then $B=C(\mathfrak{M})$.

Proof. The condition imposed in the statement of the lemma means that given two disjoint closed sets in $\mathfrak{M}$, there is an element $h \in B$ such that $h(M)$ is 1 on one set, 0 on the other set and $\|h\| \leqq K_{2}$. We may also assume that $h$ is non-negative since we may replace it by $|h|^{2}$, taking, if necessary, a bigger $K_{2}$.

Let $f$ be a non-negative function in $C(\mathfrak{M})$, define:

$$
\begin{aligned}
& P_{1}=\left\{M ; f(M) \geqq\left(1-\frac{1}{3 K_{2}}\right) \operatorname{Sup} f\right\}, \\
& P_{2}=\{M ; f(M) \leqq 1 / 2 \operatorname{Sup} f\},
\end{aligned}
$$

and let $h(M)$ be a non-negative element in $B$, of norm $\leqq K_{2}$ which is identically 1 on $P_{1}$ and vanishes on $P_{2} \cdot f_{1}(M)=\left(2 K_{2}\right)^{-1} \operatorname{Sup} f \cdot h(M)$ has the following properties: $\left\|f_{1}\right\| \leqq 1 / 2$ Sup $f, f-f_{1}$ is non-negative and $\operatorname{Sup}\left(f-f_{2}\right)<\left(1-\left(1 / 3 K_{2}\right)\right)$ Sup $f$ and the lemma follows from Lemma 1 with $K=1 / 2$ and $K_{1}=1-\left(1 / 3 K_{2}\right)$.

Definition 1. $B(P)$, where $P$ is closed in $\mathfrak{M}$, is the algebra of restrictions of $B$ to $P$ or, equivalently, the quotient algebra of $B$ by the kernel of $P$.

Definition 2. We say that $B$ is bounded in a set $V \subseteq \mathfrak{M}$ if there exists a constant $K=K(V)$ such that whenever $h$ is an idempotent in $B(P)$ with $P \subseteq V,\|h\|<K(V)$.

LEMMA 3. Let $B$ be bounded in $V_{1}$ and in $V_{2}$ where $V_{1}$ and $V_{2}$ are open in $\mathfrak{M}$. Then $B$ is bounded in every closed subset of $V_{1} \cup V_{2}$.

Proof. Let $W$ be a closed subset of $V_{1} \cup V_{2}$. We may assume $W=\mathfrak{M}$ (since we can confine our attention to $B(W)$ instead of $B$ ). There exist open sets $W_{1}, W_{2}$ satisfying: $\bar{W}_{j} \subset V_{j} ; W_{1} \cup W_{2}=\mathfrak{M}$. Since $B$ is regular it contains a function $\phi$,

$$
\phi(M)= \begin{cases}0 & M \notin W_{1}, \\ 1 & M \notin W_{2} .\end{cases}
$$

If $P$ is closed in $\mathfrak{M}, P=\left(P \cap \bar{W}_{1}\right) \cup\left(P \cap \bar{W}_{2}\right)$ and every idempotent in $B(P)$ can be obtained as $\phi h_{1}+(1-\phi) h_{2}$ where $h_{j}$ is an idempotent in $B\left(P \cap \bar{W}_{j}\right)$ and the lemma follows.

Definition 3. $B$ is bounded at a maximal ideal $M$ if there is a neighborhood $V$ of $M$ such that $B$ is bounded in $V$.

Lemma 4. Let $P$ be compact in $\mathfrak{M}$; if $B$ is bounded at every $M \in P$, there exists an open $V \supseteq P$ such that $B$ is bounded in $V$. 
This is an obvious consequence of Lemma 3.

LEMMA 5. If the idempotents of any quotient algebra of $B$ are bounded, there is at most a finite number of points in $\mathfrak{M}$ at which $B$ is not bounded.

PROOF. If there were infinitely many there would exist a sequence $\left\{M_{j}\right\}_{j=1}^{\infty}$ with disjoint neighborhoods $V_{j}$ such that $B$ would not be bounded in $V_{j}$. There would be a closed $P_{j} \leqq V_{j}$ such that $B\left(P_{j}\right)$ would contain an idempotent of norm $\geqq j$.

If $P=\bigcup P_{j}$ then $B(\bar{P})$ would not have its idempotents bounded.

The preceding proof yields actually more. We see that under the conditions of Lemma 5, there exists, for every family of disjoint open sets $\left\{V_{\alpha}\right\}$, a constant $K$ such that $K\left(V_{\alpha}\right) \leqq K$ for all but a finite number of $\alpha$ 's.

Let us now show that, under the condition of Lemma $5, B$ is bounded at every $M \in \mathfrak{M}$. We may obviously assume that there is only one point "in doubt" and denote it by $M_{0}$.

There is a neighborhood $V$ of $M_{0}$ and a constant $K$ such that every idempotent in $B(P)$, where $P \subset V$ and has $M_{0}$ as an isolated point, has norm less than $K$. (Use the same argument as in the proof of Lemma 5.) Using Lemmas 3, 4 and the assumption that $M_{0}$ was the only point at which $B$ was not known to be bounded we see that we may take $V=\mathfrak{M}$. For every closed $P$ that has $M_{0}$ as an isolated point $B(P)=C(P)$ (Lemma 2 ) and there is a constant $A$, independent of $P$, such that the norm in $B(P)$ is bounded by $A$ times the Sup norm. This implies [1, the last lemma] $B=C(\mathfrak{M})$ and we thus proved

THEOREM. If the idempotents of any quotient algebra of $B$ are bounded, $B=C(\mathfrak{M})$.

CoRollary (For Terminology see [1]). If there is a function $F(x)$ defined for $-1<x<1$ that operates in $B$ and such that

$$
F(0)=0, \quad \lim _{x \rightarrow 0} x^{-1} F(x)=\infty .
$$

Then $B=C(\mathfrak{M})$.

Proof. Use [1, Theorem 1] and the fact that $F$ operates also in any quotient algebra of $B$.

\section{REFERENCE}

1. Y. Katznelson, Sur les algèbras dont les éléments non-negatifs admettent des racines carrées, Ann. Sci. Ecole Norm. Sup. vol. 77 (1960).

UNIVERSITY OF CALIFORNIA, Berkeley, California 